\title{
Cardiopulmonary Resuscitation during COVID-19 Pandemic: Outcomes, Risks, and Protective Strategies for the Healthcare Workers and Ethical Considerations
}

\author{
Atul P Kulkarni ${ }^{1}$, Yudhyavir Singh ${ }^{2}$, Heena Garg ${ }^{3}$, Simant Jha ${ }^{4}$
}

\section{Abstract}

The crisis caused by Coronavirus disease-2019 (COVID-19) pandemic has led us to safeguard ourselves and our colleagues against transmission of this highly contagious infection, while aiming for the same goals of care. In spite of the stringent measures adopted by affected countries, rising number of healthcare workers (HCWs) are getting infected, dwindling the scarce manpower at our disposal. In the pre-COVID-19 times, cardiopulmonary resuscitation (CPR) was offered unhesitantly to all patients, who had even a slim chance of achieving return of spontaneous circulation. In COVID-19 era, CPR, due to some components being high aerosol-generating procedures (AGPs), has become high-risk procedure for the HCWs. Instead of "Primum non nocere" (first do no harm), we are forced to change to "Primum non nocere ad te" (first do no harm to yourself). The challenge is therefore to provide best possible chance of survival to deserving patients, whose COVID-19 status might be unknown, without causing harm to the HCWs. In this review, we discuss the current data regarding infected HCWs, outcomes of inhospital and out-of-hospital cardiac arrests, components of CPR which are high-risk AGPs, how to safeguard the HCWs while offering CPR, and the ethical considerations when CPR is considered, in this COVID-19 era. We wish to emphasize here that there is NO EMERGENCY in a pandemic, and time must be made for donning appropriate PPE. We feel that clear policies need to be developed by the institutions to deliver CPR to correct population, in this challenging period. Keywords: Aerosol-generating medical procedures, Cardiopulmonary resuscitation, COVID-19, Personal protective equipment, Transmission of infection.

Indian Journal of Critical Care Medicine (2020): 10.5005/jp-journals-10071-23544
\end{abstract}

\section{INTRODUCTION}

Coronavirus infections disease-2019 (COVID-19) pandemic, caused by highly contagious SARS-CoV-2 virus, has overwhelmed health infrastructure around the world, in spite of stringent measures. The healthcare workers (HCWs) dealing with COVID-19 patients directly have the highest risk of infection transmission. The WHO reported that as of April 21, 2020, over 35,000 HCWs were infected. ${ }^{1}$ The Chinese Center for Disease Control (China CDC) and Prevention found that of 44,672 (62\%) confirmed cases, 1,716 (3.8\%) were HCWs. ${ }^{2}$ In Wuhan, a very high $(63 \%, 1,080$ of 1,716$)$ proportion of HCWs were infected. Of these 247 (14.8\%) had critical infections and 5 died. ${ }^{3}$ In India, the incidence of confirmed COVID-19 cases among HCWs was $5 \% .{ }^{4}$

Cardiopulmonary resuscitation (CPR) is offered to most inpatients suffering from cardiac arrest, unless advance directives are present, or the patient has a known do-not-resuscitate (DNR) status. The reports from China, France, and Italy suggest that outcomes of COVID-19 patients, who suffer either inhospital or out-of-hospital arrest, are poor. ${ }^{5-8}$ Since many components of CPR generate aerosol, the WHO has listed CPR as an aerosol-generating procedure (AGP). ${ }^{9}$ Infected HCWs pose a "triple threat" in current circumstances, loss of a HCWs to, and addition of a new patient (the HCW) to the overburdened healthcare systems, and a source of infection to coworkers and community. ${ }^{10}$ The normal dictum "Primum non nocere" (first do no harm) needs to be altered to "Primum non nocere ad te" (first do no harm to yourself) at present.

In this review, we discuss the risk of infection transmission to the HCWs and protective measures for them. We also summarize the ethical principles in providing and withholding CPR. Finally, we suggest a protocol for preventing transmission of infection to the HCWs, during CPR (Table 1).
${ }^{1}$ Division of Critical Care Medicine, Department of Anesthesia, Critical Care and Pain, Tata Memorial Hospital, Homi Bhabha National Institute, Parel (E), Mumbai, Maharashtra, India

${ }^{2,3}$ Department of Anesthesiology, Critical Care and Pain Medicine, All India Institute of Medical Sciences, New Delhi, India

${ }^{4}$ Department of Critical Care, Pushpawati Singhania Research Institute, New Delhi, India

Corresponding Author: Atul P Kulkarni, Division of Critical Care Medicine, Department of Anesthesia, Critical Care and Pain, Tata Memorial Hospital, Homi Bhabha National Institute, Parel (E), Mumbai, Maharashtra, India, Phone: +91-9869077526, e-mail: kaivalyaak@ yahoo.co.in

How to cite this article: Kulkarni AP, Singh $Y$, Garg $H$, Jha $S$. Cardiopulmonary Resuscitation during COVID-19 Pandemic: Outcomes, Risks, and Protective Strategies for the Healthcare Workers and Ethical Considerations. Indian J Crit Care Med 2020;24(9): 868-872.

Source of support: Nil

Conflict of interest: None

\section{Outcomes of Cardiac Arrest in COVID-19 Patients Inhospital Cardiac Arrests in COVID-19 Patients}

Shao et al. reported outcomes of inhospital cardiac arrests (IHCAs) in 136 patients, during early days of COVID-19 pandemic in a Wuhan hospital. ${ }^{5}$ Most $(113,83.1 \%)$ arrests occurred in general wards and respiratory arrest was the most frequent $(119,87.5 \%)$. The commonest initial rhythm was asystole (89.7\%), followed by shockable rhythms (5.9\%) and pulseless electrical activity (PEA,

(c) The Author(s). 2019 Open Access This article is distributed under the terms of the Creative Commons Attribution 4.0 International License (https://creativecommons. org/licenses/by-nc/4.0/), which permits unrestricted use, distribution, and non-commercial reproduction in any medium, provided you give appropriate credit to the original author(s) and the source, provide a link to the Creative Commons license, and indicate if changes were made. The Creative Commons Public Domain Dedication waiver (http://creativecommons.org/publicdomain/zero/1.0/) applies to the data made available in this article, unless otherwise stated. 
Table 1: Suggested protocol for preventing transmission of infection to healthcare workers during cardiopulmonary resuscitation, in the coronavirus disease-2019 era

1. Treat all the patients as having COVID-19 infection, unless proved otherwise.

2. First don the PPE, the minimum delay causes it is OK!

3. Remember, there is no emergency in a pandemic!!!

4. Follow $A B C$ rather than $C A B$, sealing of the airway is important to prevent aerosolization.

5. Use muscle relaxant for intubations in peri-arrest conditions

6. Insert a supraglottic airway device early or intubate with a videolaryngoscope, if expertise is available, to avoid Bag-Mask ventilation

7. Use a HEPA filter between the airway and ventilating device, as soon as it is secured.

8. If already intubated, ventilate using the ventilator, at low rate (10 bpm) and low tidal volume [6 mL/kg ideal body weight (IBW)].

9. If not already ventilated, connect with ventilator as early as possible.

10. Attach closed in-line suction.

11. Maintain a closed circuit.

12. Change the person giving chest compression frequently, if possible every minute.

13. If available, use mechanical chest compression devices.

14. If the patient is in prone position, defibrillate with pads positioned anteroposteriorly or in both axillae.

15. If patients is intubated, start chest compressions in prone position, monitor $\mathrm{ETCO}_{2}$ and Systolic blood pressure (with arterial line)

16. If $\mathrm{EtCO}_{2}<10 \mathrm{~mm} \mathrm{Hg}$, turn patient supine and continue chest compressions.

17. If the patient is prone but not intubated, turn supine before starting CPR.

18. If initial rhythm was asystole, and ROSC not achieved in 30 minutes, stop CPR.

19. Decontaminate equipment used in CPR as per local institutional protocol.

4.4\%). In spite of arrests occurring in monitored patients and CPR within 1 minute, return of spontaneous circulation (ROSC) was achieved in only 18 patients. Four patients survived, while one had a favorable neurological outcome at 30 days. The reasons for the poor outcomes may be shortage of medical resources and lack of good-quality CPR. Contrary to this, in the same center in 2016 the main cause was cardiac (47.6\%), followed by other (unspecified) etiologies (20.5\%) and then respiratory (17.5\%) causes. Return of spontaneous circulation was achieved in a higher proportion of (35.5\%) patients; more (9.1\%) patients were discharged alive and many $(6.4 \%)$ had good neurological recovery. ${ }^{11}$

An earlier review of ventilated patients with pneumonia and sepsis with IHCA also had different pattern, in that arrest occurred due to cardiac reasons (50-60\%), and then respiratory insufficiency (15-40\%). ${ }^{12}$ The initial rhythm was asystole or PEA in majority (87\%) of patients, and $60 \%$ patients were on vasopressor infusions. The rate of survival to discharge was $12.5 \%$. The rate of survival with no or moderate neurological disability was $9.2 \%$, and only $6.2 \%$ had good neurological outcome. The authors suggested that till outcome data from COVID-19 patients are available, this data could be used. They suggested that universal DNR orders, COVID-19 era are not warranted.

\section{Out-of-hospital Cardiac Arrests in COVID-19 Patients}

Baldi et al. reported a higher (a 58\% increase) incidence of out-ofhospital cardiac arrests (OHCAs) during the pandemic, as compared to $2019 .^{7}$ The median time taken to provide EMS was longer and number of patients who received bystander CPR was less (by 15.6\%), which increased the incidence of deaths (by 14.9\%). The increase in the number of OHCAs was mostly due to an increase (77.4\%) in the number of patients with suspected or proven COVID-19. This points toward the poor outcome of OHCAs in COVID-19 patients. The French data are similar to the Italian data. Marijon et al. reported that the over a period of 4.5 weeks, weekly OHCA incidence doubled to 26.64 from 13.42, per million inhabitants, in the beginning of pandemic, before returning to baseline. ${ }^{8}$ The increase in OHCAs was mostly due to $30 \%$ increase in COVID-19 cases. Marijon et al. speculated that poor outcomes were caused by lockdown, behavior changes in bystanders, and an overwhelmed EMS.

\section{CPR and the Risk of Transmission of COVID-19 Infection}

Cardiopulmonary resuscitation is a complex maneuver that includes chest compression, defibrillation, and airway management, and many of these can generate aerosol. ${ }^{9}$ As studies about aerosol generation with many components are inconclusive, we need to be cautious in interpreting the results. ${ }^{13}$

\section{Chest Compressions and Defibrillation during CPR and Aerosol Deneration}

The evidence about chest compressions and defibrillation being AGPs is very weak. Tran et al. reported that the odds ratio (OR) for SARS transmission due to chest compression was 1.4 (95\% Cl $0.2-$ 11.2). ${ }^{13}$ Couper et al. said that very little evidence exists that either chest compressions or defibrillation are AGPs. ${ }^{14}$ Chest compressions might generate very small tidal volumes; however, whether this is enough to carry a significant risk of transmission of infection is unknown. ${ }^{15}$ Ott et al. photographed aerosol spread, in a simulation study during chest compressions. Mostly aerosol spread toward the person giving compressions. ${ }^{16}$ With a facemask, aerosol moved toward the patients forehead. Insertion of supraglottic airway device (SAD) with filter attached stopped the spread completely. Early insertion of SAD connected to a filter may produce a twofold benefit: treatment of hypoxia and protection of the HCWs. The European Resuscitation Council avers that both chest compressions and CPR are potential AGPs and, HCWs should don PPE during resuscitation. ${ }^{17}$ However, it may be worthwhile to try defibrillation for shockable rhythm, before donning PPE, since ROSC may occur, preventing the need for CPR. Use of mechanical chest compression devices is suggested for anticipated prolonged resuscitation. In any case, during cardiac arrest, patients will also need ventilation or their airway secured, which are more likely to generate aerosols.

\section{Aerosol Generation and Risk of Transmission of Infection during Airway Management}

Judson and Munster described how aerosol is generated in various medical procedures. ${ }^{18}$ They divided AGPs into procedures that cause patient to generate aerosols (cough during tracheal intubation (TI) without paralytic agents) and those that cause mechanical generation of aerosols (e.g., bag-mask ventilation $(B M V))$. Bag-mask ventilation (BMV) and positive pressure ventilation (PPV) generate aerosols by forcing air into the respiratory tract, though the exact mechanism is unknown. Tran 
et al. found that the pooled OR for COVID-19 transmission was 6.6 (95\% Cl 2.3-18.9) in four cohort and four case control studies. ${ }^{13}$ The OR for transmission of infection during manual ventilation was $2.8(95 \% \mathrm{Cl} 1.3-6.4)$ and after intubation was 1.3 (95\% Cl 0.5-3.2). $\mathrm{BMV}$ is the commonest method for providing breaths during CPR. Here ensuring tight mask fit is important. A two-handed grip, using $\mathrm{C}$ and $\mathrm{E}$ technique, minimizes leaks. The other advantage of SADs is that some SADs can act as conduit for intubation. ${ }^{19}$

TI allows uninterrupted chest compressions during ventilation. The most experienced operator, while interrupting chest compressions briefly, should perform TI. Use of videolaryngoscope by an experienced staff leads to fewer episodes of prolonged $(>10$ seconds) interruptions. ${ }^{20}$ American Heart Association (AHA) in its interim guidelines recommended the use of videolaryngoscope for intubation in COVID-19 patients. ${ }^{21}$ After TI, the endotracheal tube (ETT) should be connected to a ventilator circuit with a HEPA filter in the expiratory limb. The use of a closed circuit and closed in-line suction decreases the chances of aerosolization. ${ }^{22}$ If already intubated, the patient should be immediately connected to the ventilator, $\mathrm{FiO}_{2}$ changed to 1.0. The respiratory rate should be 10 bpm and the tidal volume should be $6 \mathrm{~mL} / \mathrm{kg}$ IBW. Once ROSC is achieved, ventilator settings should be reset appropriately. ${ }^{22}$

\section{Protective Measures during CPR}

During IHCA, HCWs, in their zeal, will rush to save the patient. It must be emphasized, there is NO EMERGENCY in a pandemic and time must be given to don appropriate PPE. The safety of the clinician should be the first priority!!! Losing an HCW owing to infection means losing part of valuable taskforce, and subsequently more patients. In spite of the likely delay, it is a must that we don PPE, before CPR. In a simulation-based donning/doffing study, potential biosafety breaches, which may infect HCWs if not executed properly, have been mentioned. ${ }^{23}$ Performing CPR with PPE is challenging and tiring, but you have to be cautious so that no breach occurs in PPE. Both donning and doffing of PPE should be practiced repeatedlyin accordance with standard guidelines by the WHO and the local hospital policy, along with adequate disposal of PPE. ${ }^{24}$

\section{Difficulties in Performing CPR Due to PPE}

Shao et al. described the problems (and the solutions) encountered during resuscitation. ${ }^{25}$ They recommended that the team members should wear loose-fitting clothing and pay proper attention to the tying of the mask since it can slip during CPR. If the mask still slips, someone else should take over. They suggested frequent changes (every minute) of persons performing chest compressions, or use of mechanical compression devices, since performing chest compressions while wearing PPE causes rapid fatigue. Using a positive pressure respirator hood could help in protection.

\section{Strategies to Protect the HCWs During CPR Advanced Care Planning}

Advanced care planning should be done in all patients. Mclntosh suggested that all inpatients should mandatorily have a treatment escalation plan (TEP) filled at the time of admission, to be kept updated during stay. ${ }^{26}$ There have been some calls for extreme recommendations, i.e., universal DNR orders. For example, University Hospitals Birmingham NHS Foundation Trust issued directions to doctors, not to offer CPR to patients not in the ED, unless doctors were wearing full PPE. ${ }^{27}$ A joint statement of the Belgian Society of Emergency and Disaster Medicine and the Belgian Resuscitation Council suggested that CPR be avoided when provider safety cannot be guaranteed. ${ }^{28}$ While we do not know the way COVID-19 patient will progress, we still need to evaluate these patients, before making drastic decisions.

\section{Use of Plastic Sheets as Barriers}

Allen et al. suggested using clear plastic sheets impervious to water over patients, during performance of AGPs such as BMV, TI, and CPR. ${ }^{29}$ They suggested that patients should be covered completely. Further, if the HCWs made an opening in the sheet for some procedure, it should be taped shut, as soon as possible, to prevent aerosol escape. The sheets should remain on, till the procedure is done and be removed if no HCW can be at the patient's side. The sheet should be removed slowly, while being rolled upon itself to prevent spread of fomites, and disposed of appropriately.

\section{Restricted Number of HCWs for CPR}

A minimum number of providers should be involved in CPR so that total exposure can be reduced, and also resources such as PPE can be conserved.

\section{CPR in Prone Position}

Many patients with COVID-19 develop acute lung injury or acute respiratory distress syndrome (ARDS), causing acute hypoxic respiratory failure (AHRF). Acute respiratory distress syndrome may be atypical in some patients. ${ }^{30}$ In two retrospective studies, ARDS developed in 31 and 65 patients. ${ }^{31,32}$ After the PROSEVA trial, prone ventilation is being increasingly used for severe ARDS. ${ }^{33}$ Elharrar et al. tried the prone position in COVID-19 patients with AHRF in the ward. ${ }^{34}$ Only $63 \%$ patients tolerated the prone position for $>3$ hours, and oxygenation improved in only one-fourth, which was not sustained after change to the supine position in $50 \%$ patients. An Italian study reported an improvement in oxygenation and reduction in the respiratory rate in patients on NIV in the prone position. ${ }^{35}$ The accompanying editorial was cautiously optimistic but suggested need for further research. ${ }^{36}$ The ICS, UK, suggests the prone position, in conscious patients requiring $28 \%$ or higher inspired oxygen. ${ }^{37}$

Performing CPR in a prone position poses different challenges. It will depend on whether the patient is already intubated and not. ${ }^{38}$ Prone CPR may generate higher systolic and mean arterial pressures. ${ }^{39}$ In nonintubated COVID-19 patients, CPR may be delayed due to time required to don the appropriate PPE. The UK resuscitation council recommends defibrillation before chest compressions to avoid delay in the delivery of defibrillation. The ERC guideline suggests two defibrillator pad placement options for prone patients anterior-posterior or biaxillary. ${ }^{17}$

Nonintubated prone patients will need to be turned supine for airway control. This needs at least six people, with possible risk of dislodgement of tubes and lines, causing delay in chest compressions. However the HCWs must don PPE, before doing any of this, to protect themselves. For intubated patients in the ICU, there will be no delay in CPR, because the HCWs are in full PPE. To avoid delay, CPR can be attempted in a prone position, using $\mathrm{ETCO}_{2}$ and arterial pressure waveform. During position change, the HCWs must have a clear understanding of when and how to clamp ETT, or temporarily stop ventilation, to reduce the risk. ${ }^{17,40}$

\section{Cardiac Arrest in COVID-19 Patients: Causes and Treatment}

It is of utmost importance that any reversible cause of a cardiac arrest should be identified and treated immediately before considering stopping CPR and it remains the same. Apart from the $5 \mathrm{H}^{\prime} \mathrm{s}$ and 
$5 T^{\prime} s$ that need to be identified and rectified as early as possible, ${ }^{40}$ in dealing with COVID-19 patients, 6th and 7th $\mathrm{H}$ (hypomagnesemia and hydroxychloroquine) should also be considered. ${ }^{41}$ Otherwise, the guidelines for pharmacotherapy during ACLS remain the same in COVID-19 patients except few considerations. Lignocaine use is preferred in case of arrhythmias, avoiding amiodarone, due to possible QT prolongation due to its interaction with drugs such as hydroxychloroquine and azithromycin that the patient may be receiving. ${ }^{40,41}$

\section{Post-resuscitation Care and Debriefing}

All the equipment used during CPR should be carefully handled and disposed of as per hospital policy. Once ROSC is achieved, the factors, which may have contributed to cardiac arrest, should be recognized and treated promptly. Post-resuscitation care is the same for COVID-19 patients. ${ }^{42}$ Debriefing is an effective practice for refining clinician behavior, which is associated with enhanced technique and patient outcome. ${ }^{43}$ Debriefing can be performed via video call interactions with other HCWs in the time of COVID-19.

\section{Ethical Considerations and Dilemmas}

Medical ethics is a branch of philosophy that involves recommending and defending concepts of right and wrong conduct. Ethical approaches to health care can be divided into the utilitarian (consequentialist) approach and the Kantian deontological approach. ${ }^{44,45}$ In the utilitarian approach, decisions are made to obtain the greatest benefit obtained for the greatest possible number of individuals. This may cause harm to a few patients, while its overall outcome is beneficial to a large number of individuals. In the deontological approach, the decision is made for the best outcome of the individual patient, and may or may not be good for the society. Traditionally, medical profession is deontological in nature, and is the basis of long-term bond between doctor and patient.

In the context of the COVID-19 pandemic, which approach should we adopt? The calls by certain healthcare administrators for not resuscitating patients when outcomes are likely to be poor, and may also cause harm to the HCWs, even if they are wearing $\mathrm{PPE}$, are utilitarian in nature. The same is true regarding thoughtful and appropriate allocation of ventilated beds and other resources, recently by others. ${ }^{46,47}$ This is, we think, totally justifiable in the current scenario. The infected HCWs will be out of action for a long period of time due to illness and quarantine, meaning that a trained person is not available for future patients, in the current situation where HCWs are scarce and precious resources.

\section{Conclusion}

During current COVID-19 pandemic, we must consider every collapsed patient to be a COVID-19 patient. Risk of infection to HCWs is very real and a few have died already. CPR generates aerosols, which can infect the HCWs. Efforts to maintain PPE integrity is a must during CPR. We feel that a utilitarian approach, to achieve maximum good for the greatest number, is quite sensible. In patients with multiple comorbidities, and nonreturn of ROSC, a sensible policy to either not starting or not continuing CPR should be adopted. Adequate simulation training and drills in donning and doffing of PPE for CPR is advocated. All institutes should formulate guidelines for protecting HCWs during CPR.

\section{References}

1. WHO calls for healthy, safe and decent working conditions for all health workers, amidst COVID-19 pandemic https://www.who.int/ news-room/detail/28-04-2020/. last accessed 14th June 2020.

2. Wu Z, McGoogan JM. Characteristics of and important lessons from the coronavirus disease 2019 (COVID-19) outbreak in China: summary of a report of 72314 cases from the Chinese center for disease control and prevention. JAMA 2020;323(13):1239-1242. DOI: 10.1001/ jama.2020.2648.

3. Wang D, Hu B, Hu C, Zhu F, Liu X, Zhang J, et al. Clinical characteristics of 138 hospitalized patients with 2019 novel coronavirus-infected pneumonia in Wuhan, China. JAMA 2020;323(11):1061-1069. DOI: 10.1001/jama.2020.1585.

4. Chatterjee $P$, Anand T, Singh KJ, Rasaily R, Singh R, Das $S$, et al. Healthcare workers \& SARS-CoV-2 infection in India: a casecontrol investigation in the time of COVID-19. Indian J Med Res 2020;151(5):459-467. DOI: 10.4103/ijmr.IJMR_2234_20.

5. Shao F, Xu S, Ma X, Xu Z, Lyu J, Ng M, et al. In-hospital cardiac arrest outcomes among patients with COVID-19 pneumonia in Wuhan, China. Resuscitation 2020;151:18-23. DOI: 10.1016/j. resuscitation.2020.04.005.

6. Girotra S, Tang Y, Chan P, Nallamothu BK. Survival after in-hospital cardiac arrest in critically ill patients: implications for the COVID-19 pandemic? Preprint medRxiv 2020. DOI: 10.1101/2020.04.11.20060749.

7. Baldi E, Sechi GM, Mare C, Canevari F, Brancaglione A, et al. Out-ofhospital cardiac arrest during the Covid-19 outbreak in Italy. N Engl J Med 2020;383(5):496-498. DOI: 10.1056/NEJMc2010418.

8. Marijon E, Karam N, Jost D, Perrot D, Frattini B, Derkenne C, et al. Out-of-hospital cardiac arrest during the COVID-19 pandemic in Paris, France: a population-based, observational study. The Lancet Public Health 2020;5(8):E437-E443. DOI: 10.1016/S2468-2667(20)30117-1.

9. World Health Organisation. https://www.who.int/emergencies/ diseases/novel-coronavirus-2019/technical-guidance/infectionprevention-and-control.

10. Prin $M$, Bartels K. Social distancing: implications for the operating room in the face of COVID-19. Can J Anaesth 2020;67(7):789-797. DOI: 10.1007/s12630-020-01651-2.

11. Shao F, Li CS, Liang LR, Qin J, Ding N, Fu Y, et al. Incidence and outcome of adult in-hospital cardiac arrest in Beijing, China. incidence and outcome of adult in-hospital cardiac arrest in Beijing, China. Resuscitation 2016;102:51-56. DOI: 10.1016/j. resuscitation.2016.02.002.

12. Andersen LW, Holmberg MJ, Berg KM, Donnino MW, Granfeldt A. In-hospital cardiac arrest: a review. JAMA 2019;321(12):1200-1210. DOI: 10.1001/jama.2019.1696.

13. Tran K, Cimon K, Severn M, Pessoa-Silva CL, Conly J. Aerosol generating procedures and risk of transmission of acute respiratory infections to healthcare workers: a systematic review. PLoS ONE 2012;7(4):e35797. DOI: 10.1371/journal.pone.0035797.

14. Couper K, Taylor-Phillips S, Grove A, Freeman K, Osokogu O, Court $\mathrm{R}$, et al. COVID-19 in cardiac arrest and infection risk to rescuers: a systematic review. Resuscitation 2020;151:59-166. DOI: 10.1016/j. resuscitation.2020.04.022.

15. Deakin CD, O'Neill JF, Tabor T. Does compression-only cardiopulmonary resuscitation generate adequate passive ventilation during cardiac arrest? Resuscitation 2007;75(1):53-59. DOI: 10.1016/j.resuscitation.2007.04.002.

16. Ott M, Milazzo A, Liebau S, Jaki C, Schilling T, Krohn A, et al. Exploration of strategies to reduce aerosol-spread during chest compressions: a simulation and cadaver model. Resuscitation 2020;152:P192-P198. DOI: 10.1016/j.resuscitation.2020.05.012.

17. https://www.erc.edu/covid/European Resuscitation Council COVID19 Guidelines/ last accessed 14th June 2020.

18. Judson SD, Munster VJ. Nosocomial transmission of emerging viruses via aerosol-generating medical procedures. Viruses 2019;11(10):940. DOI: $10.3390 / v 11100940$. 
19. Panchal AR, Berg KM, Cabañas JG, Kurz MC, Link MS, Del Rios M, et al. American Heart Association focused update on systems of care: dispatcher-assisted cardiopulmonary resuscitation and cardiac arrest centers: an update to the American Heart Association guidelines for cardiopulmonary resuscitation and emergency cardiovascular care. Circulation 2019;140(24):e895-e903. DOI: 10.1161/ CIR.0000000000000733.

20. Newell C, Grier S, Soar J. Airway and ventilation management during cardiopulmonary resuscitation and after successful resuscitation. Crit Care 2018;22(1):90. DOI: 10.1186/s13054-018-2121-y.

21. Edelson DP, Sasson C, Chan PS, Atkins DL, Aziz K, Becker LB, et al. Interim guidance for basic and advanced life support in adults, children, and neonates with suspected or confirmed COVID-19: from the emergency cardiovascular care committee and get with the guidelines ${ }^{\oplus}$-resuscitation adult and pediatric task forces of the American Heart Association in collaboration with the American Academy of Pediatrics, American Association for Respiratory Care, American College of Emergency Physicians, the Society of Critical Care Anesthesiologists, and American Society of.... Circulation 2020;141(25):e933-e943. DOI: 10.1161/CIRCULATIONAHA.120.047463.

22. Phua J, Weng L, Ling L, Egi M, Lim CM, Divatia JV, et al. Intensive care management of coronavirus disease 2019 (COVID-19): challenges and recommendations. Lancet Respir Med 2020;8(5):506-517. DOI: 10.1016/S2213-2600(20)30161-2.

23. Muñoz-Leyva F, Niazi AU. Common breaches in biosafety during donning and doffing of protective personal equipment used in the care of COVID-19 patients. Can J Anaesth 2020;67(7):900-901. DOI: 10.1007/s12630-020-01648-x.

24. World Health Organisation. https://www.who.int/csr/resources/ publications/putontakeoffPPE/en/ last accessed 13th June 2020.

25. Shao F, Sun P, Tang Z. Cardiopulmonary resuscitation of inpatients with severe COVID-19 pneumonia: the Wuhan experience. Resuscitation 2020;152:95-96. DOI: 10.1016/j.resuscitation.2020.05.009.

26. McIntosh L. Can the COVID-19 crisis strengthen our treatment escalation planning and resuscitation decision making? Age Ageing 2020(4):afaa083. DOI: 10.1093/ageing/afaa083.

27. Mahase E, Kmietowicz Z. Covid-19: doctors are told not to perform CPR on patients in cardiac arrest. BMJ 2020;368:m1282. DOI: 10.1136/ bmj.m1282.

28. https://www.brc-rea.be/wp-content/uploads/2020/03/Ethicaldecision-making-in-emergencies_COVID19_22032020_final.pdf/. lastaccessed on 10th June 2020.

29. Allen B, Gardner C, O'Neill C, Gibbs M. Use of drape/patient covering during potentially aerosolizing procedures. Am J Emerg Med 2020. S0735-6757(20)30343-0 10.1016/j.ajem.2020.05.007.

30. Gattinoni L, Coppola S, Cressoni M, Busana M, Rossi S, Chiumello D. Covid-19 does not lead to a "typical" acute respiratory distress syndrome. Am J Respir Crit Care Med 2020;201(10):1299-1300. DOI: 10.1164/rccm.202004-1052LE.

31. Zhou F, Yu T, Du R, Fan G, Liu Y, Liu Z, et al. Clinical course and risk factors for mortality of adult inpatients with COVID-19 in Wuhan, China: a retrospective cohort study. Lancet 2020;395(10229):10541062. DOI: 10.1016/S0140-6736(20)30566-3.

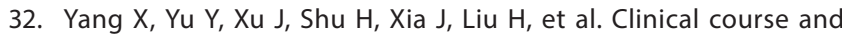
outcomes of critically ill patients with SARS-CoV-2 pneumonia in
Wuhan, China: a single-centered, retrospective, observational study. Lancet Respirat Med 2020;8(5):475-481. DOI: 10.1016/S2213-2600(20) 30079-5.

33. Guérin C, Reignier J, Richard JC, Beuret P, Gacouin A, Boulain T, et al. Prone positioning in severe acute respiratory distress syndrome. $\mathrm{N}$ Engl J Med 2013;368(23):2159-2168. DOI: 10.1056/NEJMoa1214103.

34. Elharrar X, Trigui Y, Dols AM, Touchon F, Martinez S, Prud'homme E, et al. Use of prone positioning in nonintubated patients with COVID19 and hypoxemic acute respiratory failure. JAMA 2020(22):e208255. DOI: 10.1001/jama.2020.8255.

35. Sartini C, Tresoldi M, Scarpellini P, Tettamanti A, Carcò F, Landoni $G$, et al. Respiratory parameters in patients with COVID-19 after using noninvasive ventilation in the prone position outside the intensive care unit. JAMA 2020(22):e207861. DOI: 10.1001/jama. 2020.7861.

36. Telias I, Katira BH, Brochard L. Is the prone position helpful during spontaneous breathing in patients with COVID-19? JAMA 2020;323(22):2265-2267. DOI: 10.1001/jama.2020.8539.

37. https://www.ficm.ac.uk/sites/default/files/prone_position_in_adult_ critical_care_2019.pdf/ last accessed 13th June 2020.

38. Barker J, Koeckerling D, West R. A need for prone position CPR guidance for intubated and non-intubated patients during the COVID-19 pandemic. Resuscitation 2020;151:135-136. DOI: 10.1016/ j.resuscitation.2020.04.029.

39. Mazer SP, Weisfeldt M, Bai D, Cardinale C, Arora R, Ma C, et al. Reverse CPR: a pilot study of CPR in the prone position. Resuscitation 2003;57(3):279-285. DOI: 10.1016/s0300-9572(03)00037-6.

40. Link MS, Berkow LC, Kudenchuk PJ, Halperin HR, Hess EP, Moitra VK, et al. Part 7: adult advanced cardiovascular life support. 2015 American Heart Association guidelines update for cardiopulmonary resuscitation and emergency cardiovascular care. Circulation 2015;132(18 suppl 2):S444-S464. DOI: 10.1161/CIR.0000000000000261.

41. Kapoor A, Pandurangi U, Arora V, Gupta A, Jaswal A, Nabar A, et al. Cardiovascular risks of hydroxychloroquine in treatment and prophylaxis of COVID-19 patients: a scientific statement from the Indian heart rhythm society. Indian Pacing Electrophysiol J 2020;20(3):117-120. DOI: 10.1016/j.ipej.2020.04.003.

42. Pothiawala S. Post-resuscitation care. Singapore Med J 2017;58(7): 404-407. DOI: 10.11622/smedj.2017060.

43. Couper K, Perkins GD. Debriefing after resuscitation. Curr Opin Crit Care 2013;19(3):188-194. DOI: 10.1097/MCC.0b013e32835f58aa.

44. Mandal J, Ponnambath DK, Parija SC. Utilitarian and deontological ethics in medicine. Trop Parasitol 2016;6(1):5-7. DOI: 10.4103/22295070.175024 .

45. Garbutt G, Davies P. Should the practice of medicine be a deontological or utilitarian enterprise? J Med Ethics 2011;37(5): 267-270. DOI: 10.1136/jme.2010.036111.

46. Biddison EL, Faden R, Gwon HS, Mareiniss DP, Regenberg AC, SchochSpana $M$, et al. Too many patients... a framework to guide statewide allocation of scarce mechanical ventilation during disasters. Chest 2019;155(4):848-854. DOI: 10.1016/j.chest.2018.09.025.

47. Emanuel EJ, Persad G, Upshur R, Thome B, Parker M, Glickman A, et al. Fair allocation of scarce medical resources in the time of COVID-19. N Engl J Med 2020;382(21):2049-2055. DOI: 10.1056/NEJMsb2005114. 